\title{
Csaba Földes (Hg.): Res humanae proverbiorum et sententiarum. Ad honorem Wolfgangi Mieder. Tübingen: Narr, 2004 (405 s.)
}

Unter diesem latinisierenden Titel erschien 2004 die vom ungarischen Germanisten und Phraseologen Csaba Földes herausgegebene Festschrift, die den 60. Geburtstag des allseits beliebten, anerkannten und außerordentlich produktiven Parömiologen, Phraseologen et al. Wolfgang Mieder markiert. Eine aktuelle Werkliste des Jubilars befindet sich unter

http://www.uvm.edu/\%7Egrdept/?Page=Wolfgang_Mieder.html

Der Gewürdigte, der an der Universität Vermont in den USA lehrt, ist nicht zuletzt für seine Herausgabe der Zeitschrift Proverbium bekannt. Es gibt aber kaum ein phraseologisches Gebiet, zu dem der Jubilar nicht entscheidend beigetragen hat. So viel zum Empfänger selbst, der die Festschrift an der EUROPHRAS-Tagung in Basel im August 2004 entgegennahm (EUROPHRAS: http: //www.europhras.unizh.ch). So wie die abgebildeten Wortschatzeinheiten des bekannten (bauern-)brueghelschen Gemäldes "Die niederländischen Sprichwörter" (1562), das die Titelseite der Festschrift schmückt, eine bunte Mischung aus Phraselogie, Parömiologie, Monolexik u. a. m. ist, so ist auch der Inhalt der Festschrift sehr heterogen, was mit der breiten Forschungstätigkeit Wolfgang Mieders in gutem Einklang steht. Obwohl die Parömiologie - Schwerpunkt der Forschung Mieders - sehr stark vertreten ist, werden auch Phraseme im Allgemeinen, geflügelte Worte sowie andere Probleme behandelt. Auch die theoretischen und methodologischen Zugänge sind vielfältig; es gehören u. a. historische, texttypologische, translatorische, kontrastive, literarische, kulturelle und genderwissenschaftliche dazu. Anstatt dass ich alle Beiträge bespreche, was kaum zu bewältigen wäre, erwähne ich zunächst bloß die einzelnen Arbeiten. Danach behandle ich eine Auswahl der Beiträge eingehender, die mir aus dem einen oder anderen Grund besonders erwähnenswert oder diskussionswürdig erscheinen.

Albertsson/Korhonen untersuchen die Sprichwort-Kodifizierung in deutschschwedischen Wörterbüchern, Arora geht auf die Rolle von Sprichwörtern in spanischer Narration ein, Chlosta/Grzybek behandeln das oft diskutierte Problem der Bekanntheit von Parömien, Dǔrčo untersucht das slowakische "proverbial minimum" empirisch, Eismann erörtert phraseologische Figurenkennzeichnung im Drama, Fiedler beschäftigt sich mit Sprichwörtern in DDR-Comics, Erzébet Forgács geht auf die Rolle der Phraseologie in "kreativen Texten" ein, Tamás Forgács diskutiert unikale Komponenten in ungarischen Phraseologismen, Lindahl untersucht parömiologische Beziehungen zwischen Märchen und "Everyday Conversation of a Kentucky Mountain Family", McKenna untersucht die Funktion von Sprichwörtern bei Tolstoj, Mokienko würdigt die Rolle 
Mieders bei der Erforschung der slawischen Phraseologie, Norrick beschäftigt sich mit Hyperbolik und ECFs ("Extreme Case Formulations") in englischen Sprichwörtern, Elisabeth Piirainen zeigt, wie Linguisten in den Titeln ihrer Arbeiten intertextuell mit phraseologischen "prefab"-Teilen arbeiten, Ilpo Piirainen geht auf historische Phraseologie anhand der Luther-Schrift "Von der Freiheit eines Christenmenschen" ein, Raders demonstriert die Aktualität von Goethe-Zitaten, Rothkegel untersucht die Funktion negierter Phraseme, SkogSödersved schreibt über Phraseologismen in der Textsorte Horoskop, Litovkina untersucht "Anti-Proverbs in the Language Classroom", Trokhimenko erörtert den Zusammenhang von Sprichwörtern und Gender-Sozialisierung im Mittelhochdeutschen, Ueda greift am Beispiel des Deutschen und Japanischen den beliebten Vergleich von Tierbezeichnungen in der Phraseologie wieder auf, Voigt nimmt historische Bezeichnungen europäischer Sprichwörter unter die Lupe, und Wirrer betrachtet die niederdeutsche Übersetzung der schleswig-holsteinischen Landesverfassung aus der phraseologischen Warte.

Im Folgenden wird also auf eine kleine Auswahl der oben nicht genannten Beiträge näher eingegangen:

\section{Sprichwörter und kognitive Metapherntheorie}

In einer sehr interessanten Studie wendet Kispál das Lakoff/Johnsonsche Instrumentarium auf die Parömiologie an. Der Aufsatz behandelt eine Menge theoretischer Fragen und liefert Material aus vielen verschiedenen europäischen Sprachen. Dabei sind manche der dort vorgestellten "Konzepte" wie etwa MENSCHEN SIND UNBELEBTE GEGENSTÄNDE (S. 131) m. E. problematisch: Zwar werden Sprichwörter wie neue Besen kehren gut; der Apfel fällt nicht weit vom Stamm und stille Wasser sind tief (auch) mit Bezug auf Menschen verwendet, aber das heißt wohl nicht unbedingt, dass die Sprecher dann eine solche "primitive" Metapher konzeptuell gespeichert haben? Vgl. auch die „Konzepte“ GLAUBEN SIND KINDER wegen man soll das Kind nicht mit dem Bade ausschütten und IDEEN SIND SPEISEN auf Grund von too many cooks spoil the broth (dän. for mange kokke fordcerver maden). Denn sie basieren offensichtlich häufig auf einem sehr kleinen Inventar an Sprichwörtern und können deshalb kaum als wirklich produktiv gelten. Ich frage mich deshalb, was der theoretische Wert einer solchen konzeptuellen Metapher ist, die nicht falsifizierbar ist und für Prädikationen kaum taugt? Der Artikel demonstriert m.E. das kognitivistische Grundproblem, dass gewisse auffindbare Muster in der Lexik sehr rasch zu kognitiven Realitäten erhoben werden, obwohl es doch eine sehr gewagte und letztendlich sich selbst bestätigende Annahme ist. Kíspal erwähnt auch die bekannte kognitivistische Kritik an der "traditionellen" semasiologi- 
schen Lexikographie: Sie würde zu sehr am Lexem festhalten statt endlich den "Konzepten" den ihnen gebührenden Platz einzuräumen. Dabei fehlt m. E. oft eine lexikographische Theoriebildung: "die Verwendung von Sprichwörtern zu fördern", wie es bei Kíspal heißt, ist wohl eine recht periphere lexikographische Funktion. Und es ist kaum einsehbar, dass mit Angaben unter dem Lemma Liebe wie LIEBE IST EIN WEG und LIEBE IST EINE REISE in der Sprachproduktion wirklich erfolgreich umgegangen werden kann. M. E. müssten die Kognitivisten experimentell nachweisen, dass solche Angaben wirklich eine produktive Hilfe darstellen, bevor die aus meiner Sicht recht radikale Forderung gemacht wird, die Wörterbücher mögen die Vorschläge der kognitiven Semantik übernehmen. Trotzdem ist der Beitrag Kispáls sehr lesenswert.

Auch Lewandowska/Antos beschäftigen sich mit dem Problem von Sprichwörtern und Kognitivik, wenn auch bei ihnen der Begriff"Kultur" den Schwerpunkt bildet, weshalb sie ihren Zugang "kultur-kognitiv" nennen. Ihre Annahme, dass beim Verstehen des Sprichworts keine Rosen ohne Dornen über das "Konzept" AUCH BEI DEN SCHÖNSTEN SACHEN GIBT ES (KLEINERE) NACHTEILE - ich würde statt "Konzept" einfach von einer semantischen Paraphrase reden -, "ein spezifisches Wissen über die kulturelle Bedeutung von Rosen als Symbol der Liebe/der Wertschätzung bzw. über Dornen, die in der christlichen Tradition als Symbol des Leidens gelten" vorausgesetzt sei, scheint mir empirisch falsch zu sein: Beim Lernen des Sprichworts keine Rose ohne Dornen spielt m. E. einzig seine Gebrauchsregel eine Rolle. Man kann das Sprichwort sowohl adäquat verwenden als auch verstehen, ohne dass man seinen "kulturellen" Hintergrund kennt, und außerdem lernt man wohl meistens erst die Etymologie (wenn überhaupt), nachdem man das Sprichwort bereits erfolgreich produzieren und rezipieren kann. M. E. halten die Autoren Synchronie und Diachronie nicht konsequent genug auseinander. Dabei handelt es sich offenbar um ein häufiges Problem des Kognitivismus, der aus meiner Sicht nicht immer zwischen linguistisch Relevantem und Epiphänomenen unterscheidet.

\section{"Das Wörterbuch zur österreichischen Phraseologie"}

ist eine Projektvorstellung von Ernst/Peyerl. Ausgehend von der Feststellung, dass die nicht-bundesdeutsche (Standard-)Phraseologie lexikographisch nicht zufrieden stellend beschrieben ist, haben die beiden Autoren 2003 ein Projekt initiiert, das diesem Defizit abhelfen soll. Dass auch innerhalb der Standardsprache viele Unterschiede zwischen der Phraseologie des deutschen, schweizerischen und österreichischen Deutsch existieren, ist in der Tat ein wenig beachteter Fakt. So sind z. B. zentrale phraseologische Helvetismen wie Anfang/Mitte/Ende Jahr/ Monat/Woche/...; in (den) Ausgang gehen (, ausgehen um zu feiern'); etw ${ }_{D}$ einen Riegel schieben (,etw verhindern'); am selben Strick ziehen (,am selben Strang 
ziehen'); gebranntes Wasser (,Schnaps') im DUDEN (2003) nicht aufgenommen (Farø 2004). Das Pionierwerk Ammon et al. (2004) wird zwar auch hier für einige Abhilfe sorgen. Es handelt sich bei dieser Arbeit aber nicht um ein Spezialwörterbuch für Phraseologie, deswegen ist die Initiative Ernst/Peyerls begrüßenswert. Die beiden Autoren gehen von der plausiblen theoretischen Grundlage aus, dass das österreichische Deutsch eine eigene, (zumindest wissenschaftlich) gleichberechtigte Standardvarietät darstellt, neben dem Standarddeutsch der Schweiz und Deutschlands. Als "österreichischer Phraseologismus" - nahe liegend wäre auch die Bezeichnung "Austrophraseologismus" - ist nach den Verfassern "eine Redewendung dann anzusprechen, wenn sie von seit ihrer Geburt im österreichischen Staatsgebiet lebenden Personen als in der Standardsprache bekannt UND gebräuchlich angegeben wird und wenn sie signifikante Unterschiede zur bundesdeutschen oder schweizerdeutschen Standardsprache aufweist" (82). Es handelt sich also um ein Differenzwörterbuch, und streng genommen nicht um ein "Wörterbuch der österreichischen Phraseologie", vgl. die beiden expliziten Differenzwörterbücher Meyer (1989) und Ebner (1998). Das Projekt basiert auf einer umfassenden neuen Empirie, darunter Zeitungen, Belletristik und Alltagstextsorten wie z. B. Kochbüchern. Die Autoren rechnen mit der Erfassung von nicht weniger als 50.000 österreichischen Phrasemen, dabei werden diese, falls relevant, auch mit Angaben zum Vorkommen in den jeweiligen Bundesländern sowie mit verschiedenen soziolinguistischen Spezifizierungen versehen. Das Ergebnis wird sowohl in elektronischer als auch in einer Druckfassung vorliegen, dabei wird das Wörterbuch "nur" etwa 10-15.000 Phraseme umfassen. Leider geben die Autoren keine Hinweise darauf, wie sie das Problem der Kontrolle des tatsächlichen "Differenzcharakters" der Phraseme lösen. In Farø (2004) wurde eine recht einfache - wenn auch nicht 100 $\%$ sichere - operationelle Methode zur Lösung dieses Problems vorgeschlagen. Ernst/Peyerl nennen in ihrem Artikel einige Beispiele österreichischer Phraseologismen, darunter (1) jmd. ist (wieder) am/auf den Dampfer ('jd. ist wieder gesund und munter'), (2) Deutsches Eck, das sich in Österreich nicht in erster Linie auf den Rhein-Mosel-Zusammenlauf in Koblenz bezieht, sondern auf den Südostgipfel Bayerns, (3) etw. hängt jm beim (Dtl.: zum) Hals heraus, (4) von der Leber weg sprechen (bundesdt. frei von der ...), (5) einen Knödel im Hals haben (bundesdt.: Kloß), (6) Bruderschaft trinken (bundesdt. Brüderschaft). Wenn man diese Phraseme in Ammon et al. (2004) nachschlägt und anhand der Methode Farøs (2004) kontrolliert, so stellen sich 2 Tendenzen heraus: Die Praseme sind nicht alle im neuen Variantenwörterbuch kodifiziert (immerhin (3) und (4)). Damit hätte das neue Projekt bereits seinen Wert bewiesen. Nur: Stichproben bei Google (jeweils 50) zeigen, dass es mit dem rein österreichischen Charakter der Phraseme hapern könnte: (wieder) am/auf dem Dampfer sein ist z. B. nur in $10 \%$ der Fälle auf österreichischen Homepages zu finden, sonst tauchen die 
Belege auf deutschen Websites auf. Bei von der Leber weg sprechen waren gerade $8 \%$ der Homepages österreichisch, $10 \%$ dagegen schweizerisch und der Rest deutsch. Auch mit Bruderschaft trinken kommen keine eindeutigen Zahlen. Bei einen Knödel im Hals haben sieht es dagegen besser aus: Hier stammen 76 $\%$ der Belege aus österreichischen Homepages (bei $20 \%$ deutschen), womit wir uns in der Terminologie Farøs einem absoluten Austriazismus zumindest nähern. Vielleicht sind dies Indizien dafür, dass wir es entweder 1) eher mit allgemeinen süddeutschen Phrasemen zu tun haben oder 2) mit Phrasemen, die zwar in Österreich überproportional häufig vorkommen und damit als relative, aber nicht als absolute Phraseologismen gerechnet werden können (vgl. Farø 2004). Ich bin mir die Probleme dieser Methode bewusst, jedoch meine ich, dass sie zumindest einen Hinweis auf Relevantes geben kann.

\section{Der Phraseologiebegriff}

Levin-Steinmann geht noch einmal dem definitorischen Problem nach, was überhaupt unter „Phraseologie“ verstanden werden soll. Die Autorin stellt fest, dass sich v. a. viele neuere Arbeiten um diese Begriffsbestimmung nicht kümmern. Sie liefert dann eine eigene Definition, deren wesentliche Merkmale Mehrwortcharakter, Bezeichnungsanomalie (Ergebnis eines "conceptual shifts"), semantisch-strukturelle Festigkeit sind (156). Das Hauptgewicht wird dabei auf die beiden ersten Merkmale gelegt. Dass dies die "Phraseologismen i. w. S." erfassen würde, dem kann ich nicht ganz zustimmen, da doch damit sämtliche kompositionell gebildete Kollokationen ausgeschlossen würden (Geld abheben, das Fahrrad schieben), die aus meiner und vieler anderer Sicht zur Phraseologie gehören und durchaus im Rahmen explizit phraseologischer Fragestellungen behandelt werden (vgl. das Vorwort des vorliegenden Bands). Die Verfasserin stellt den Begriff "Phraseologisierung" in die Nähe der Grammatikalisierung, indem sie Phraseme mit präpositionaler Funktion ("sekundäre Präpositionen" nach Hentschel) wie mit Hilfe und in Richtung untersucht. Levin-Steinmann betont, dass "die" Phraseologie-Konzeption vom Blickwinkel der jeweiligen Untersuchung abhängt und deshalb kaum definitiv festgelegt werden kann. Das ist vielleicht etwas übertrieben formuliert, aber auch nicht ganz falsch.

\section{Pump aerobics-Phraseologie}

Kuiper/Lodge untersuchen Phraseologie als Mittel zur "controlling the body", indem sie "the specialised phraseology" von Aerobic ("pump instructor talk") zu ihrem Untersuchungsgegenstand machen. Vier Trainerinnen wurden während ihrer Arbeit auf Band aufgenommen; die Autoren stellen dabei Folgendes fest: Was während einer Trainingsstunde von der Trainerin "to cue their routines" gesagt wird, ist "almost entirely formulaic" (146f.), z. B.: lower your arms 
to the sides!; backs nice and straight!; feet shoulder with apart, team!; here we go!; all right team!; hold it there!; two down, two up!; give me [X]! - $\mathrm{zu}$ letzterem Beispiel (einer Phraseoschablone), vgl. Farø (2005). Darüber hinaus interessiert die beiden Autoren die Phraseologie als Höflichkeitsträger im Rahmen der face-Theorie Brown/Levinsons. Es handelt sich dabei meistens um so genannte Routineformeln (Coulmas 1981); dabei sind Routineformeln nicht exklusiv phraseologisch (vgl. Farø 2003a), wie auch einige der eigenen Beispiele der Autoren demonstrieren (u. a. ok!). Es stellt sich heraus, dass die Trainerinnen sehr unterschiedliche Höflichkeitsstrategien an den Tag legen, von der "team player"-Strategie (all right!, give me [X]!) bis zu gezielten "face attacks": can you feel this?; What are you? A bunch of chickens?) Die Verfasser schlussfolgern, dass die spezifischen Bedingungen des Aerobic-Trainings (u. a. Zeitdruck, ständige Wiederholungen) eine formelhafte Sprache generieren, die situationell adäquat, weil ökonomisch, ist. Neue "trainees" haben dabei häufig Schwierigkeiten, dieser (halbwegs idiolektalen) "oral formulaic tradition" zu folgen. Kuiper/Lodge betonen, dass "pump aerobics-Phraseologie" nur ein Beispiel unter vielen möglichen "phraseologies of body control" ist.

\section{Japanische Sprichwörter}

Diese materialreiche Studie von Paczolay zeigt, dass die Quellen japanischer Sprichwörter, abgesehen von einigen autochtonen Beispielen, v. a. chinesischen Ursprungs ist. Hinzu kommen in neuerer Zeit viele Entlehnungen, zu meinem Erstaunen zunächst aus dem Deutschen, später dann v. a. aus dem Englischen. Der Beitrag ist nicht zuletzt auch deswegen interessant, weil er die relative Übersetzbarkeit von Parömiologie demonstriert, auch wenn es sich um linguistisch voneinander entfernte Sprachen wie Japanisch vs. SAE-Sprachen handelt. Gleichzeitig zeigt die Studie den Nutzen von Literalisierungen, wenn es darum geht, sprachübergreifende Kategorien wie „Sprichwort“ zu definieren, was m. E. nicht ausgeschlossen werden sollte: Zumindest scheint es möglich, parömiologische "Kandidaten" zu falsifizieren. Des Japanischen unkundig, muss ich mich auf Paczolays Literalisierungen verlassen. Dabei ist auf der Grundlage der Definition von Farø (2003b: 41) leicht feststellbar, dass es sich bei seinem Material keineswegs nur um Sprichwörter handelt. Zwar ist das Kriterium (3) Farøs (Lexikalisierung) für mich nicht kontrollierbar, die beiden anderen Kriterien (1: Textcharakter) und (2: generalisierende - oder besser: generische (Jakobsen 2005) - Aussage) dagegen sehr wohl. Bei vielen Beispielen Paczolays fehlen beide Merkmale offensichtlich, die ich als unverzichtbar betrachte, soll "Sprichwort" als wissenschaftliche Kategorie eindeutig vom Gros der phraseologischen Subkategorien unterschieden werden. Somit können - wenn wir von den Literalisierungen Paczolays ausgehen (Hepburn-Transliteralisierung) -, 
uo /no/ ki ni noboru ga gotoshi ("like a fish climbing a tree"); ishi no ue ni mo sannen ("he can endure) three years on top of a stone"); hari no ana kara tenjo nozoku ("to look at the sky through the eye of a needle"); shi ni uma ni (mo) hari (sasu) ("to apply a needle <of acupuncture $>$ (even) to a dead horse"); ebi de tai wo tsuru ("to catch a sea-bream with a shrimp"); wa-ga ta he mizu wo hiku ("to draw water to his own rice-field") und e ni kaita mochi ("painted rice-cakes" [sie stillen den Hunger nicht])

kaum als Sprichwörter akzeptiert werden. Es handelt sich wohl dagegen um Idiome, weil sie nur potenzielle und keine fertigen Texte sind und sie stellen auch keine generischen Aussagen dar. Dagegen scheinen Beispiele wie umoregi ni hana (ga) saku ("flower blossoms on fossil wood") und jigoku no sata mo kane shidai ("money rules even in the court of hell") genuine Sprichwörter zu sein - wenn sie wohlgemerkt lexikalisiert sind. Die Falsifikationsmöglichkeit scheint zu belegen, dass „Sprichwort“ doch eine interlinguale (universale?) linguistische Kategorie sein könnte.

Insgesamt enthält die Festschrift eine reiche Sammlung an Aufsätzen zu vielen wichtigen und interessanten Themen und Fragestellungen der aktuellen Phraseologieforschung. Dass kritisable Einzelheiten zu vermerken sind, die selbstverständlich teilweise theorieabhängig sind, mindert den Wert des Bandes keineswegs. Somit stellt er eine adäquate Würdigung des großen Parömiologen, Humanisten et al. Wolfgang Mieder dar.

\section{Literatur}

Ammon, Ulrich et al. 2004: Variantenwörterbuch des Deutschen. Die Standardsprache in Österreich, der Schweiz und Deutschland sowie in Liechtenstein, Luxemburg, Ostbelgien und Südtirol. Berlin/New York: Walter de Gruyter.

Coulmas, Florian 1981: Routine im Gespräch. Zur pragmatischen Fundierung der Idiomatik. (= Linguistische Forschungen 29). Wiesbaden: Akademische Verlagsgesellschaft Athenaion.

DUDEN 2003 = Duden Universalwörterbuch auf CD-ROM. Mannheim: Dudenverlag.

Ebner, Jakob 1998: Wie sagt man in Österreich? Wörterbuch des österreichischen Deutsch. 3., vollst. überarb. Aufl. Mannheim etc.: Bibliographisches Institut.

Farø, Ken 2003a: Det ligger lige på tungen. Den danske fraseologi. In Mål + Mcele 3/2003, 19-27.

Farø, Ken 2003b: Ordsprog i nutidsdansk. Funktioner og problemer. In Danske Studier 98, 38-64.

Farø, Ken 2004: Verbildete, verbeamtete und überalterte Deutschschweizer? Das Schweizerhochdeutsche im Wörterbuch und sonst. In Geoffrey Williams/Sandra Vessier 
(Hg.): Proceedings of the Eleventh EURALEX International Congress. S. 879-884. Bretagne: UBS.

Farø, Ken (2005): Én gang vind og skæv terrorist... Fraseoskabeloner og fraseologisk reduplikation. In Danske Studier.

Jakobsen, Lisbeth Falster (2005): Det er sundt med daglig motion. Om frasemer og generiske ytringer i tekster. In Jens Cramer/Mette Kunøe/Ole Togeby (Hg.): Teorien om alt. Og andre artikler om sprog og filosofi. S. 135-147. Århus: Wessel og Huitfeldt. Meyer, Kurt (1989): Wie sagt man in der Schweiz? Mannheim: Dudenverlag.

Ken Farø 